\title{
Inverse Shortest Path in a Graph with Rough Edge Weights
}

\author{
Sagarika Biswal \\ Department of Mathematics \\ College of Engineering and Technology \\ Bhubaneswar, India-751003
}

\author{
S. P. Mohanty \\ Department of Mathematics \\ SOA University \\ Bhubaneswar, India-751030
}

\begin{abstract}
The inverse shortest path problem occurs mostly in reconstruction type of problems where, minimum modifications of the edge weights of a network are made to make a predetermined path to be shortest. In this paper, initially the edge weights are taken as rough variables which, are based on the subjective estimation of the experts. Then these rough weights are approximated by normal uncertain variables and an uncertain programming model has been developed. Further, the uncertain programming model is transformed into a deterministic counterpart which can be solved by any standard method.
\end{abstract}

\section{Keywords}

Shortest path, Inverse shortest path, Uncertain variable, Rough variable, Linear programming problem.

\section{INTRODUCTION}

In inverse shortest path problem in a connected weighted graph, any pre-determined path from origin to destination is to be made a shortest path after modification of weights of the edges where the total modification shall be minimum. This problem has been studied by many researchers due to its different reconstruction problems. The traffic network improvement problem is a typical inverse shortest path problem. Considering the travel time between two localities of a city in a particular path, the number of public transportation system should be modified or width of the existing roads are to be increased to facilitate smooth traffic so as reduce the travel time.

Burton and Toint [1] formulated the inverse shortest path problem for the first time where they have used $l_{2}$ norm to measure the modification of the weights. Xu and Zhang [2] discussed the general structure of the feasible set of weights in the inverse shortest path problem and showed the relation between inverse shortest path problem and the minimal cutset problem. Zhang and Liu [3] formulated an inverse shortest path problem as a special linear programming problem under $\mathrm{l}_{1}$ norm. $\mathrm{Hu}$ and Liu [4] also developed an algorithm to solve the inverse shortest path problem using $l_{1}$ norm

In most of the cases, the edge weights are assumed to be deterministic which does not hold in practical applications. For example, the traveling time in a road network may be affected by weather conditions, number of vehicles in the road, heavy accidents, road block due to police checking etc which are uncertain and cannot be predicted before. In these cases, some researchers believed that the nondeterministic nature can be handled with probability theory or fuzzy theory. However, when no samples are available to estimate a probability distribution in the nondeterministic environment, the views of some domain experts are used to evaluate the belief degree that each event will occur. In order to deal with the belief degree rationally, the uncertainty theory was developed by Liu [5] in 2007 and subsequently applied the theory to model the shortest path problem.

So far as inverse shortest path problem is concerned, the problem is not adequately investigated in uncertain environment. Zhou et al. [6] in 2014 investigated the problem of inverse shortest path considering the edge weights as linear uncertain variables. They have showed that when the edge weights are independent uncertain variables with regular distribution, then the uncertain inverse shortest path problem can be formulated into a deterministic programming model.

In this work, the edge weights are taken as rough variables initially and subsequently approximated by uncertain normal variables. The model so developed reduces to a deterministic linear programming problem model.

The rest of the paper is organized as follows: In Section 2 classical deterministic inverse shortest path problem is discussed and some basic concepts of uncertainty theory, uncertain variables, and rough variables are presented. In Section 3, uncertain inverse shortest path problem is formulated. The equivalent crisp model is discussed in Section 4. In Section 5, one numerical example is presented for illustration. Finally, the conclusion is given in Section 6.

\section{PRELIMINARIES}

In this section, the classical inverse shortest path problem is revived and some notions and results of uncertain variable are rough variable are given which shall be used to handle uncertain inverse shortest path.

\subsection{Classical Inverse shortest path problem}

In the classical inverse shortest path problem, new weights are assigned to the edge of a graph such that a predetermined path is the shortest path from the origin to the destination with respect to the new edge weights and the modification is minimum.

Let $\mathrm{G}=(\mathrm{V}, \mathrm{E})$ be a connected graph with vertex set $\mathrm{V}=$ $\left\{\mathrm{v}_{1}, \mathrm{v}_{2}, \mathrm{v}_{3}, \ldots, \mathrm{v}_{\mathrm{n}}\right\}$ and the edge set $\mathrm{E}=\{1,2,3, \ldots, \mathrm{m}\}$

For each edge $i \in E$, there is an original weight $c_{i}$ as well as new weights $x_{i}$ after a modification. $P_{0}$ is the predetermined path from origin to destination which is required to be the shortest path with respect to the new weights.

The inverse shortest path problem can be formulated as follows. 


$$
\left\{\begin{array}{l}
\min \sum_{i=1}^{n}\left|x_{i}-c_{i}\right| \\
\text { subject to } \\
\text { i) } P_{0} \text { is the shortest path } \\
\text { with respect to } x_{i}{ }^{\prime} s \\
\text { ii) } x_{i} \geq 0 \text { for } i \in E
\end{array}\right.
$$

\subsection{Uncertainty Theory}

B. Liu [5], [7] has developed uncertainty theory which is considered as a new approach to deal with indeterminacy factors when there is a lack of observed data. In this section, some basic concepts of uncertainty theory has been reviewed which shall be used in this paper.

\subsubsection{Uncertainty measure}

Let $\mathrm{L}$ be a $\delta$ - algebra on a nonempty set $\Gamma$. A set function $\mathrm{M}: \mathrm{L} \rightarrow[0,1]$ is called an uncertain measure if it satisfies the following axioms.

Axiom 1: (Normality axiom) $\mathrm{M}(\Gamma)=1$ for the universal set $\Gamma$

Axiom 2: (Duality axiom) $\mathbf{M}(\Lambda)+\mathbf{M}\left(\Lambda^{\mathrm{c}}\right)=1$ for every event $\Lambda$

Axiom 3: (sub additive axiom) For every countable sequence of events $\Lambda_{1}, \Lambda_{2}, \ldots$. we have

$\mathbf{M}\left\{\bigcup_{\mathrm{i}=1}^{\infty} \Lambda_{\mathrm{i}}\right\} \leq \sum_{i=1}^{\infty} \mathbf{M}\left(\Lambda_{\mathrm{i}}\right)$

The triplet $(\Gamma, L, M)$ is called an uncertainty space.

Axiom 4: (Product measure) Let $\left(\Gamma_{k}, L_{k}, M_{k}\right)$ be uncertainty spaces for $\mathrm{k}=1,2 \ldots \ldots$. The product uncertain measure is an uncertain measure satisfying

$$
\mathbf{M}\left\{\prod_{k=1}^{\infty} \Lambda_{k}\right\}=\bigcap_{k=1}^{\infty} \mathbf{M}\left(\Lambda_{k}\right)
$$

where, $\Lambda_{k}$ an arbitrary chosen events for $L_{k}$ for $\mathrm{k}=$ $1,2 \ldots \ldots$ respectively.

\subsubsection{Uncertain variable}

An uncertain variable $\xi$ is essentially a measurable function from an uncertainty space to the set of real numbers. Let $\xi$ be an uncertain variable. Then the uncertainty distribution of $\xi$ is defined as $\phi(\mathrm{x})=M\{\xi \leq x\}$ for any real number $\mathrm{x}$.

An uncertain variable $\xi$ is called linear if it has linear uncertainty distribution $\mathrm{L}(\mathrm{a}, \mathrm{b})$

$$
\phi(\mathrm{x})= \begin{cases}0 & \text { if } \mathrm{x} \leq \mathrm{a} \\ \frac{\mathrm{x}-a}{b-a} & \text { if } \mathrm{a} \leq \mathrm{x} \leq \mathrm{b} \\ 1 & \text { if } \mathrm{x}>\mathrm{b}\end{cases}
$$

An uncertain variable $\xi$ is called normal if it has a normal uncertainty distribution

$$
\phi(x)=\left(1+\exp \left(\frac{\pi(e-x)}{\sqrt{3} \sigma}\right)\right)^{-1} \quad \text { for } x \in R
$$

Normal uncertainty distribution is denoted by $\mathrm{N}(\mathrm{e}, \sigma)$, where e and $\sigma$ are real numbers with $\sigma>0$ are mean and standard deviation respectively.

An uncertain distribution $\phi$ is said to be regular if its inverse function $\phi^{-1}(\alpha)$ exists and is unique for each $\alpha \in(0,1)$.

The linear uncertainty distribution $\mathrm{L}(\mathrm{a}, \mathrm{b})$ is regular and its inverse uncertainty distribution is

$$
\phi^{-1}(\alpha)=(1-\alpha) a+(\alpha) b
$$

The normal uncertainty distribution $\mathrm{N}(\mathrm{e}, \sigma)$ is also regular and its inverse uncertainty distribution is

$$
\phi(x)=e+\frac{\sigma \sqrt{3}}{\pi} \ln \frac{\alpha}{1-\alpha}
$$

\subsection{Rough variable}

The concept of rough variable is introduced by Liu [7] as uncertain variable. The following definitions are based on Liu [7].

Definition 1 : Let $\Lambda$ be a non empty set, A be $\sigma$ algebra of subsets of $\Lambda, \Delta$ be an element in A, and $\pi$ be a non negative, real- valued, additive set function on A. The quadruple $(\Lambda, \Delta, A, \pi)$ is called a rough space.

Definition 2: A rough variable $\xi$ on the rough space $(\Lambda, \Delta, A, \pi)$ is a measurable function from $\Lambda$ to the set of real numbers $\mathfrak{R}$ such that for every Borel set $\mathrm{B}$ of $\mathfrak{R}$, we have $\{\lambda \in \Lambda \mid \xi(\lambda) \in \mathbf{B}\} \in A$.

Then the lower and upper approximation of the rough variable $\xi$ are defined as follows

$$
\begin{aligned}
& \bar{\zeta}=\{\zeta(\lambda) \mid \lambda \in \Lambda\} \quad \text { (Upper approximation) } \\
& \zeta=\{\zeta(\lambda) \mid \lambda \in \Delta\} \quad \text { (Lower approximation) }
\end{aligned}
$$

Definition 3: ([a , b], [c , d]) with $\mathrm{c} \leq \mathrm{a}<\mathrm{b} \leq \mathrm{d}$ is a rough variable, where $\xi(\lambda)=\lambda$ from the rough space to the set of real numbers and

$\Lambda=\{\lambda \mid c \leq \lambda \leq d\}$ and $\Delta=\{\lambda \mid a \leq \lambda \leq b\}$ , A is the Borel algebra on $\Lambda, \pi$ is the Lebesgue measure.

Definition 4: Let $(\Lambda, \Delta, A, \pi)$ be a rough space. Then the upper and lower trust of event $\mathrm{A}$ is defined by

where, $a$ and $b$ are real numbers with $a<b$. 
$\operatorname{Tr}(\mathrm{A})=\frac{\pi\{\mathrm{A}\}}{\pi\{\Lambda\}} \quad$ and $\quad \operatorname{Tr}(\mathrm{A})=\frac{\pi\{\mathrm{A} \cap \Delta\}}{\pi\{\Lambda\}}$

The trust of the event $\mathrm{A}$ is defined as

$\operatorname{Tr}(\mathrm{A})=\frac{1}{2}(\operatorname{Tr}(\mathrm{A})+\mathrm{T} \overline{\mathbf{r}}(\mathrm{A}))$

Definition 5: Let $\xi_{1}, \xi_{2}$ be rough variables defined on the rough space $(\Lambda, \Delta, A, \pi)$. Then their sum and product are defined as

$$
\begin{aligned}
& \left(\xi_{1}+\xi_{2}\right)(\lambda)=\xi_{1}(\lambda)+\xi_{2}(\lambda) \\
& \left(\xi_{1} \cdot \xi_{2}\right)(\lambda)=\xi_{1}(\lambda) \cdot \xi_{2}(\lambda)
\end{aligned}
$$

Definition 6: Let $\xi$ be rough variables defined on the rough space $(\Lambda, \Delta, A, \pi)$ and $\alpha \in(0,1]$ then

$$
\xi_{\text {sup }}(\alpha)=\sup \{\mathrm{r} \mid \operatorname{Tr}\{\xi \geq r\} \geq \alpha\}
$$

is called $\alpha$-optimistic value of $\xi$.

$$
\xi_{\text {inf }}(\alpha)=\inf \{r \mid \operatorname{Tr}\{\xi \leq r\} \geq \alpha\}
$$

is called $\alpha$-pessimistic value of $\xi$.

Definition 7: The trust distribution $\phi:[-\infty, \infty] \rightarrow[0,1]$ of a rough variable $\xi$ is defined by

$\Phi(\mathrm{x})=\operatorname{Tr}\{\lambda \in \Lambda \mid \xi(\lambda) \leq \mathrm{x}\}$

Definition 8: The trust density function $f: \mathrm{R} \rightarrow[0, \infty)$ of a rough variable $\xi$ is a function such that $\mathrm{f}(\mathrm{x})=\int_{-\infty}^{\infty} \phi(y) d y$ holds for all $\mathrm{x} \in(-\infty, \infty)$ , where $\phi$ is trust distribution of $\xi$.

Definition 9: Let $\xi$ be rough variables defined on the rough space $(\Lambda, \Delta, A, \pi)$. The expected value of $\xi$ is defined

$\mathrm{E}(\xi)=\int_{0}^{\infty} \operatorname{Tr}\{\xi \geq r\} d r-\int_{-\infty}^{0} \operatorname{Tr}\{\xi \leq r\} d r$

If $\xi=([\mathrm{a}, \mathrm{b}],[c, d])$ be a rough variable such that $\mathrm{c}$

$\leq \mathrm{a}<\mathrm{b} \leq \mathrm{d}$, then the trust distribution $\phi(\mathrm{x})=$ $\operatorname{Tr}\{\xi \leq x\}$ is

$$
\phi(\mathrm{x})= \begin{cases}\mathrm{O} & \text { if } \mathrm{x} \leq \mathrm{c} \\ \frac{\mathrm{x}-c}{2(d-c)} & \text { if } \mathrm{c} \leq \mathrm{x} \leq \mathrm{a} \\ \frac{[(\mathrm{b}-\mathrm{a})+(\mathrm{d}-\mathrm{c})] x+2 a c-a d-b c}{2(b-a)(d-c)} & \text { if } \mathrm{a} \leq \mathrm{x} \leq \mathrm{b} \\ \frac{\mathrm{x}+\mathrm{d}-2 \mathrm{c}}{2(d-c)} & \text { if } \mathrm{b} \leq \mathrm{x} \leq \mathrm{d} \\ 1 & \text { if } \mathrm{x} \geq \mathrm{d}\end{cases}
$$

And the trust density function is defined as

$f(x)= \begin{cases}\frac{1}{2(d-c)} & \text { if } c \leq x \leq a \text { or } b \leq x \leq d \\ \frac{1}{2(b-c)}+\frac{1}{2(d-c)} & \text { if } a \leq x \leq b \\ 0 & \text { otherwise }\end{cases}$

Definition 9: Expected value of a rough variable

Let the trust distribution $\varphi$ of a rough variable $\xi$ exists, Then the expected value or mean of $\xi$ is defined as $E[\zeta]=\int_{-\infty}^{\infty} x \phi(x) d x$, provided the integral exists.

Definition 10: Variance of a rough variable

If $\xi$ is a rough variable with finite expected value $\mathrm{E}[\xi]$, then the variance of $\xi$ is defined as

$\mathrm{V}[\xi]=\mathrm{E}\left[(\xi-\mathrm{E}[\xi])^{2}\right]$

\section{UNCERTAIN INVERSE SHORTEST PATH PROBLEM}

Let $\mathrm{G}=(\mathrm{V}, \mathrm{E})$ be a connected graph with vertex set $\mathrm{V}=\{$ $\left.\mathrm{v}_{1}, \mathrm{v}_{2}, \mathrm{v}_{3}, \ldots, \mathrm{v}_{\mathrm{n}}\right\}$ and the edge set $\mathrm{E}=\{1,2,3, \ldots, \mathrm{m}\}$. Let for each edge $i \in E$, there is a weight $\xi_{i}$ which is an uncertain variable.

Suppose that $\mathrm{P}_{0}$ is the predetermined path from origin to the destination which is required to be the shortest path after modification of the weights.

Then the length of any path $\mathrm{P}$ is defined as $T_{p}(\xi)=\sum_{i \in p} \xi_{i}$, which is also an uncertain variable being the sum of uncertain variables.

Let $\psi_{p}$ be the uncertain distribution of the path length $T_{p}$. In order to formulate the inverse shortest path problem on an uncertain graph, uncertain $\alpha$-shortest path is defined as follows.

A path $\mathrm{P}_{0}$ is called an uncertain $\alpha$-shortest path from the origin to the destination if

$$
\begin{aligned}
& \min \left\{\bar{T} / m\left\{T_{p_{0}}(\xi) \leq \bar{T}\right\} \geq \alpha\right\} \\
& \quad \leq \min \left\{\bar{T} / m\left\{T_{p}(\xi) \leq \bar{T}\right\} \geq \alpha\right\}
\end{aligned}
$$

for all paths $\mathrm{p}$ from origin to destination, where $\alpha$ is the pre-determined confidence level. 
The condition (2) can be rewritten as

$$
\psi_{p_{0}}^{-1}(\alpha) \leq \psi_{p}^{-1}(\alpha)
$$

Where, $\psi_{p_{0}}^{-1}(\alpha)$ and $\psi_{p}^{-1}(\alpha)$ are the inverse uncertainty distributions of path lengths $\mathrm{T}_{\mathrm{Po}_{0}}$ and $\mathrm{T}_{\mathrm{P}}$ respectively.

For the purpose of defining the inverse shortest path problem on an uncertain graph with uncertain edge weights $\xi_{\mathrm{i}}, \mathrm{i} \in \mathrm{E}$, we assume that there is a parameter $\mathrm{c}_{\mathrm{i}}$ on each edge $i \in E$, which shall be modified to $x_{i}$ after adjustment. The edge weights $\xi_{\mathrm{i}}$ are assumed to be related to this parameter. Thus, for each edge $\mathrm{i} \epsilon \mathrm{E}$, there is an original weight $\xi_{\mathrm{i}}\left(\mathrm{c}_{\mathrm{i}}\right)$ as well as a new weight $\xi_{\mathrm{i}}\left(\mathrm{x}_{\mathrm{i}}\right)$.

Given a path $\mathrm{P}_{0}$ from the origin to the destination, the uncertain inverse shortest path problem is to find a new parameters $x_{i}$ such that $P_{0}$ becomes an uncertain $\alpha$-shortest path with respect to the new weights $\xi_{\mathrm{i}}\left(\mathrm{x}_{\mathrm{i}}\right), \mathrm{i} \in \mathrm{E}$ and total change in the parameter $\sum_{i=1}^{n}\left|x_{i}-c_{i}\right|$ is minimum.

Hence, the uncertain inverse shortest path problem can be formulated as follows.

$$
\left\{\begin{array}{l}
\min \sum_{i=1}^{n}\left|x_{i}-c_{i}\right| \\
\text { subject to } \\
\text { i) } \psi_{P_{0}}^{-1}\left(x_{j}, \alpha\right) \leq \psi_{P}^{-1}\left(x_{j}, \alpha\right), \quad P \in \Omega \\
\text { ii) } x_{i} \geq 0 \quad \text { for } \quad i \in E
\end{array}\right.
$$

where, $\alpha$ is the predetermined confidence level and $\Omega$ is the set of all paths from origin to the destination.

\section{CRISP EQUIVALENT MODEL}

Considering the edge weights $\xi_{\mathrm{i}}$ as independent uncertain variables with regular distribution $\varphi_{\mathrm{i}}, \quad \mathrm{i} \in \mathrm{E}$, for any path $\mathrm{P}$ $\epsilon \Omega$, the inverse uncertainty distribution of the path length $\mathrm{T}_{\mathrm{p}}(\xi)$ is

$$
\psi_{p}^{-1}(\alpha)=\sum_{i \in p} \phi_{i}^{-1}(\alpha)
$$

Hence, the crisp equivalent model of (3) can be formulated as

$$
\left\{\begin{array}{l}
\min \sum_{i=1}^{n}\left|x_{i}-c_{i}\right| \\
\text { subject to } \\
\text { i) } \sum_{i \in P_{0}} \phi_{i}^{-1}\left(x_{i}, \alpha\right) \leq \sum_{j \in P} \phi_{j}^{-1}\left(x_{j}, \alpha\right), P \in \Omega \\
\text { ii) } x_{i} \geq 0 \quad \text { for } \quad i \in E
\end{array}\right.
$$

In this work, we have used the uncertain variable as rough variable of the form

$$
\left(\left[x_{i}-d, x_{i}+d\right],\left[x_{i}-2 d, x_{i}+2 d\right]\right.
$$

Further, we purpose the following theorem to reformulate the problem.

Theorem 1 For the rough variable ([a , b], [c , d]), the mean is $(a+b+c+d) / 4$ and

variance $=\left(a^{2}+b^{2}+c^{2}+d^{2}+a b+c d-6 e^{2}\right) / 6$, where $e$ is the mean

Proof: The trust distribution of $\xi$ is

$$
\phi(x)=\left\{\begin{array}{lrl}
0 & \text { if } & x \leq c \\
\frac{x-c}{2(d-c)} & \text { if } & c \leq x \leq a \\
\frac{[(b-a)+(d-c)] x+2 a c-a d-b c}{2(b-a)} & (d-c) \\
& \text { if } & a \leq x \leq b \\
\frac{x+d-2 c}{2(d-c)} & \text { if } & b \leq x \leq d
\end{array}\right.
$$

The trust density function $\mathrm{f}(\mathrm{x})$ of $\xi$ is $\varphi^{\prime}(\mathrm{x})$.

So $\mathrm{f}(\mathrm{x})$ is given by

$$
\begin{aligned}
f(x) & = \begin{cases}\frac{1}{2(d-c)} & \text { if } c \leq x \leq a, b \leq x \leq d \\
\frac{1}{2(b-a)}+\frac{1}{2(d-c)} & \text { if } \quad a \leq x \leq b \\
0 & \text { otherwise }\end{cases} \\
E(\xi) & =\int_{-\infty}^{\infty} x f(x) d x \\
& =\int_{c}^{a} \frac{x}{2(d-c)} d x+\int_{a}^{b}\left[\frac{1}{2(b-a)}\right. \\
& =(a+b+c+d) / 4=e
\end{aligned}
$$

$$
\begin{aligned}
\operatorname{Var}(\xi)= & E(\xi-E(\xi))^{2} \\
= & \int_{c}^{a} \frac{(x-e)^{2}}{2(d-c)} d x+\int_{a}^{b}\left[\frac{1}{2(b-a)}+\frac{1}{2(d-c)}\right](x-e)^{2} d x \\
& \quad+\int_{b}^{d} \frac{(x-c)^{2}}{2(d-c)} d x \\
= & \left(a^{2}+b^{2}+c^{2}+d^{2}+a b+d c-6 e^{2}\right) / 6
\end{aligned}
$$

From the above theorem, considering the rough variable as $\left(\left[\mathrm{x}_{\mathrm{i}}-\mathrm{d}, \mathrm{x}_{\mathrm{i}}+\mathrm{d}_{\mathrm{i}}\right],\left[\mathrm{x}_{\mathrm{i}}-2 \mathrm{~d}_{\mathrm{i}}, \mathrm{x}_{\mathrm{i}}+\mathrm{d}_{\mathrm{i}}\right]\right)$, the mean $=\mathrm{e}_{\mathrm{i}}=\mathrm{x}_{\mathrm{i}}$ and $\mathrm{SD}=\sigma_{\mathrm{i}}=\sqrt{5} \mathrm{~d}_{\mathrm{i}}$.

Now, the rough variable is approximated by the uncertain normal variable $\xi_{\mathrm{i}}$ with mean $\mathrm{x}_{\mathrm{i}}$ and SD is $\sqrt{5} \mathrm{~d}_{\mathrm{i}}$

Hence,

$\phi_{i}^{-1}\left(x_{i}, \alpha\right)=x_{i}+\frac{\sqrt{15} d_{i}}{\pi} \ln \left(\frac{\alpha}{1-\alpha}\right)$ 
So, the model (5) can be reformulated as

$$
\left\{\begin{array}{l}
\min \sum_{i=1}^{n}\left|x_{i}-c_{i}\right| \\
\text { subject to } \\
\text { i) } \sum_{i \in P_{0}} x_{i}+\frac{\sqrt{15} d_{i}}{\pi} \ln \left(\frac{\alpha}{1-\alpha}\right) \\
\quad \leq \sum_{j \in P} x_{i}+\frac{\sqrt{15} d_{j}}{\pi} \ln \left(\frac{\alpha}{1-\alpha}\right), \in \Omega \\
\text { ii) } x_{i} \geq 0 \text { for } i \in E
\end{array}\right.
$$

Further, by introducing two auxiliary variables $\mathrm{x}_{\mathrm{i}}^{+}$and $\mathrm{x}_{\mathrm{i}}{ }^{-}$, the model can further be simplified, where

$$
x_{i}^{+}=\left\{\begin{array}{llc}
x_{i}-c_{i} & \text { if } & x_{i} \geq c_{i} \\
0 & \text { if } & x_{i}<c_{i}
\end{array}\right\}
$$

and

$$
x_{i}^{-}=\left\{\begin{array}{llc}
0 & \text { if } & x_{i} \geq c_{i} \\
c_{i}-x_{i} & \text { if } & x_{i}<c_{i}
\end{array}\right\}
$$

So, $\left|x_{i}-c_{i}\right|=x_{i}^{+}+x_{i}^{-}$

$$
\begin{gathered}
x_{i}=x_{i}^{+}-x_{i}^{-}+c_{i} \\
\mathrm{x}_{\mathrm{i}}^{+} \geq 0 \text { and } \quad 0 \leq \mathrm{x}_{\mathrm{i}}^{-} \leq \mathrm{c}_{\mathrm{i}} .
\end{gathered}
$$

So, the model (7) can be transformed to

$$
\left\{\begin{array}{l}
\text { Min } \sum_{i=1}^{m}\left(x_{i}^{+}+x_{i}^{-}\right) \\
\text {subject to } \\
\text { i) } \sum_{i \in P_{0}}\left(x_{i}^{+}-x_{i}^{-}+c_{i}+\frac{\sqrt{15} d_{i}}{\pi} \ln \left(\frac{\alpha}{1-\alpha}\right)\right) \\
\leq \sum_{j \in P}\left(x_{j}^{+}-x_{j}^{-}+c_{j}+\sqrt{15} \frac{d_{j}}{\pi} \ln \left(\frac{\alpha}{1-\alpha}\right), \quad P \in \Omega\right. \\
\text { ii) } \quad x_{i}^{+}>0, \quad i \in E \\
\text { iii) } \quad 0 \leq x_{i}^{-} \leq c_{i}, \quad i \in E
\end{array}\right.
$$

The model (9) is a deterministic linear programming problem which can be solved using any standard method.

\section{NUMERICAL EXAMPLE}

In this section, one numerical example is taken to implement the proposed model. In the following figure (Fig-1), one connected graph is considered having six vertices and ten edges. The path $\mathrm{a}-\mathrm{b}-\mathrm{e}-\mathrm{f}$ is taken as the required path to be converted to be the shortest path after modification of the weights. The level of confidence $\alpha$ is taken as 0.9 . The table- 1 gives the uncertain edge weights of the edges. The mean, SD and inverse uncertain normal distribution are given in the table- 2 .

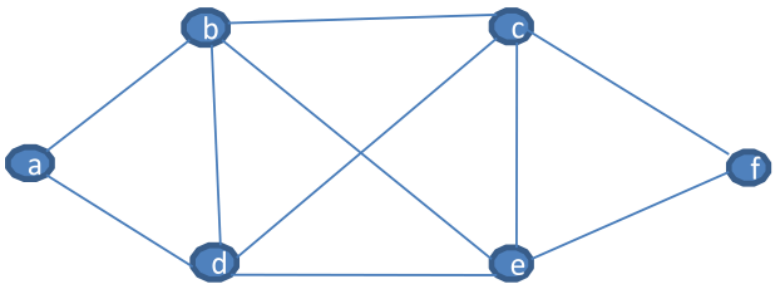

Fig-1(Network)

Table-1 (Rough edge weights)

\begin{tabular}{llll}
\hline Edge & $\begin{array}{l}\text { Edge } \\
\text { No }\end{array}$ & $\begin{array}{l}\text { Original } \\
\text { parameter } \\
\left(\mathrm{c}_{\mathrm{i}}\right)\end{array}$ & Uncertain edge weight $\left(\xi_{\mathrm{i}}\left(\mathrm{x}_{\mathrm{i}}\right)\right)$ \\
\hline$(\mathrm{a}, \mathrm{b})$ & 1 & 30 & $\left(\left[\mathrm{x}_{1}-10, \mathrm{x}_{1}+10\right],\left[\mathrm{x}_{1}-20, \mathrm{x}_{1}+20\right]\right)$ \\
\hline$(\mathrm{b}, \mathrm{d})$ & 2 & 50 & $\left(\left[\mathrm{x}_{2}-10, \mathrm{x}_{2}+10\right],\left[\mathrm{x}_{2}-20, \mathrm{x}_{2}+20\right]\right)$ \\
\hline$(\mathrm{b}, \mathrm{c})$ & 3 & 40 & $\left(\left[\mathrm{x}_{3}-10, \mathrm{x}_{3}+10\right],\left[\mathrm{x}_{3}-20, \mathrm{x}_{3}+20\right]\right)$ \\
\hline$(\mathrm{c}, \mathrm{e})$ & 4 & 80 & $\left(\left[\mathrm{x}_{4}-10, \mathrm{x}_{4}+10\right],\left[\mathrm{x}_{4}-20, \mathrm{x}_{4}+20\right]\right)$ \\
\hline$(\mathrm{c}, \mathrm{f})$ & 5 & 50 & $\left(\left[\mathrm{x}_{5}-10, \mathrm{x}_{5}+10\right],\left[\mathrm{x}_{5}-20, \mathrm{x}_{5}+20\right]\right)$ \\
\hline$(\mathrm{a}, \mathrm{d})$ & 6 & 40 & $\left(\left[\mathrm{x}_{6}-10, \mathrm{x}_{6}+10\right],\left[\mathrm{x}_{6}-20, \mathrm{x}_{6}+20\right]\right)$ \\
\hline$(\mathrm{d}, \mathrm{e})$ & 7 & 90 & $\left(\left[\mathrm{x}_{7}-10, \mathrm{x}_{7}+10\right],\left[\mathrm{x}_{7}-20, \mathrm{x}_{7}+20\right]\right)$ \\
\hline$(\mathrm{d}, \mathrm{c})$ & 8 & 60 & $\left(\left[\mathrm{x}_{8}-10, \mathrm{x}_{8}+10\right],\left[\mathrm{x}_{8}-20, \mathrm{x}_{8}+20\right]\right)$ \\
\hline$(\mathrm{b}, \mathrm{e})$ & 9 & 70 & $\left(\left[\mathrm{x}_{9}-10, \mathrm{x}_{9}+10\right],\left[\mathrm{x}_{9}-20, \mathrm{x}_{9}+20\right]\right)$ \\
\hline$(\mathrm{e}, \mathrm{f})$ & 10 & 40 & $\left(\left[\mathrm{x}_{10}-10, \mathrm{x}_{10}+10\right],\left[\mathrm{x}_{10}-20, \mathrm{x}_{10}+20\right]\right)$ \\
\hline & & &
\end{tabular}

Table-2 (Normal approximation of edge weights)

\begin{tabular}{cccc}
\hline Edge No. & mean & SD & $\Phi^{-1}\left(\mathrm{x}_{\mathrm{i}}, \alpha\right)$ \\
\hline 1 & $\mathrm{x}_{1}$ & $10 \sqrt{5}$ & $\mathrm{x}_{1}+27.1$ \\
\hline 3 & $\mathrm{x}_{2}$ & $10 \sqrt{5}$ & $\mathrm{x}_{2}+27.1$ \\
\hline 4 & $\mathrm{x}_{3}$ & $10 \sqrt{5}$ & $\mathrm{x}_{3}+27.1$ \\
\hline 5 & $\mathrm{x}_{4}$ & $10 \sqrt{5}$ & $\mathrm{x}_{4}+27.1$ \\
\hline 6 & $\mathrm{x}_{5}$ & $10 \sqrt{5}$ & $\mathrm{x}_{5}+27.1$ \\
\hline 7 & $\mathrm{x}_{6}$ & $10 \sqrt{5}$ & $\mathrm{x}_{6}+27.1$ \\
\hline 8 & $\mathrm{x}_{7}$ & $10 \sqrt{5}$ & $\mathrm{x}_{7}+27.1$ \\
\hline 9 & $\mathrm{x}_{8}$ & $10 \sqrt{5}$ & $\mathrm{x}_{8}+27.1$ \\
\hline 10 & $\mathrm{x}_{9}$ & $10 \sqrt{5}$ & $\mathrm{x}_{9}+27.1$ \\
\hline
\end{tabular}

So, the model (9) is transformed to

$\operatorname{Min} \sum_{i=1}^{m}\left(x_{i}^{+}+x_{i}^{-}\right)$

subject to

i) $\quad \sum_{i \in P_{0}}\left(x_{i}^{+}-x_{i}^{-}+c_{i}+27.1\right)$

$\leq \sum_{j \in P}\left(x_{j}^{+}-x_{j}^{-}+c_{j}+27.1\right), \quad P \in \Omega$

ii) $x_{i}^{+}>0, \quad i=1,2, \ldots, 10$

iii) $0 \leq x_{i}^{-} \leq c_{i}, \quad i=1,2, \ldots, 10$

This becomes deterministic linear programming model which gives the optimal solution as 
$\mathrm{x}_{\mathrm{i}}^{+}=\{0,0,20,0,0,0,0,0,0,0\}$

$\mathrm{x}_{\mathrm{i}}{ }^{-}=\{0,0,0,0,0,0,0,0,0,0\}$

$x^{*}=\{30,50,60,80,50,40,90,60,70,40\}$

The total modification is 20 which is minimum.

\section{CONCLUSION}

In this problem, the uncertain variables are taken as rough variables. The mean and standard deviation of each rough variable are obtained. Then the rough variables are approximated by the normal uncertain variables with the mean and standard deviation of the rough variables. The uncertain inverse shortest path problem is reduced to a deterministic linear programming model which can be solved by any standard method. In this case, in the rough variables deviations from $x_{i}$ are symmetrical. In case of non-symmetrical deviation, the variance of the rough variable shall not be independent of $\mathrm{x}_{\mathrm{i}}$, rather a linear expression of $\mathrm{x}_{\mathrm{i}}$. So the standard deviation of the rough variable shall be of the form $\sqrt{A x_{i}+B}$ for some constant A and B. So, the model can be transformed to non-linear programming problem which needs further investigation.

\section{ACKNOWLEDEGMENT}

The authors wish to thank the referees for their valuable suggestions for suitable modification of the paper.

\section{REFERENCES}

[1] Burton, D., and Toint, P. L., 1992. On an instance of the inverse shortest path problem. Mathematical programming, 53(1-3), 45-61.
[2] Xu, S. J., and Zhang, J. Z., 1995. An inverse problem of the weighted shortest path problem. Japan J. of industrial and Applied Mathematics, 12(1), 47-59.

[3] Zhang, J. Z., and Liu, Z. H., 1999. A further study on inverse linear programming problems, J. Computational and Applied Mathematics, 106(2), 345-359.

[4] Hu, Z. Q., and Liu, Z. H., 1998. A strongly polynomial algorithm for the inverse shortest arborescence problem, Discrete Applied Mathematics, 82(1-3), 135-154.

[5] Liu, B., 2007. Uncertainty theory, Springer-Verlag, Berlin.

[6] Zhou, J., Yang, F., and Wang, K.., 2014. An inverse shortest path problem on an uncertain graph, Journal of Networks, 9(9), 2353-2359.

[7] Liu, B., 2004. Uncertainty Theory: An introduction to its axiomatic foundation, Springer-Verlag, Berlin.

[8] Zhang, J. Z., Ma, Z. F., and Yang, C., 1995. A column generation method for inverse shortest path problem, Mathematical Methods of Operations Research, 41(3), 347-358.

[9] Liu, B., 2010. A branch of mathematics for modelling human uncertainty, Springer-Verlag, Berlin

[10] Liu, B., 2009. Some research problems in uncertainty theory, Journal of Uncertain Systems, 3(1), 3-10 